\title{
Dossier Évolution et créationnisme Dépasser les oppositions et aborder la téléologie en questionnant la notion de vie
}

\author{
François Calatayud, Marie-Line Maublanc \\ Éthologues, CEFS, INRA, 31326 Castanet Tolosan, France
}

Dans une des contributions à ce forum, Françoise Fridlansky et Jean-Claude Mounolou (2007) situent l'un des principaux problèmes liés au débat créationnisme/théorie synthétique de l'évolution dans le champ de la privation de liberté : enrôlé malgré lui dans un camp, le chercheur voit sa liberté directement menacée par un choix qui n'en est pas vraiment un. «On n'est pas libre quand on n'est rien, on est libre quand on est ce qu'on n'est rien, on est libre quand on est ce qu'on a choisi d'être ", disait Merleau-Ponty (1997). Ainsi, cette liberté ne consiste pas à pouvoir faire n'importe quoi, mais à pouvoir s'engager en choisissant ce que l'on veut être et sur quelle voie mettre ses pas. Peut-être est-ce là que se situe le propre de la science, ce qui devrait la démarquer des autres manières d'interpréter le monde, comme la religion. Une des pistes empruntées dans le débat qui nous anime consiste à marquer l'opposition entre la science et le créationnisme. Cette étape est probablement nécessaire, mais peut rencontrer aussi quelques limites. Notamment, poser que la science est une entreprise unifiée, purement rationnelle et fonctionnant par falsifications d'hypothèses successives est quelque chose de très discutable, comme ont pu le mettre en évidence historiens et sociologues des sciences (Canguilhem, 1965a et b ; Prigogine et Stengers, 1979 ; Latour, 1995 ; Pestre, 2006). Si l'approche créationniste peut se targuer d'être une entreprise unifiée sous un dogme, c'est bien la chance de la science d'apparaître comme un concert d'opinions, de vues, de sensibilités différentes. Le "propre de la science » est quelque chose d'assez flou, et le réduire à une production d'hypothèses que l'on vérifie expérimentalement suivant une méthode bien définie va parfois même

Auteur correspondant :

F. Calatayud, Francois.Calatayud@toulouse.inra.fr à l'encontre de ce qu'elle peut être, comme Feyerabend (1988) a pu le présenter, et qui plus est lorsque l'on parle d'évolution. On pourrait également faire référence au travail d'histoire et de philosophie des sciences de Georges Canguilhem, notamment dans son étude de la théorie cellulaire (Canguilhem, 1965b). Le premier de ces deux auteurs a pu mettre en évidence que les règles méthodologiques ne conditionnent pas nécessairement le succès scientifique, voire l'empêche; le deuxième a montré que les théories ne procèdent pas des faits mais de théories antérieures auxquelles elles sont reliées par les faits. Cette diversité est le plus souvent une chance qui donne justement sa dynamique à la science. Pour Platon, dans le Théétète, le propre de la science est quelque chose de difficile à cerner, qui n'est ni sensation ni production d'opinions vraies accompagnées ou non d'une définition. Le propre de la science semble alors plus se cacher dans son étymologie, dans le $\lambda o \gamma o \sigma$ et dans la confrontation des points de vue. De fait, les tentatives de poser en dogmes ou certitudes des pans de connaissances entraînent un certain aveuglement. Ainsi, l'énonciation par Crick (1958 et 1970) du « dogme central de la biologie moléculaire » a entraîné par la suite une forte cécité de la part de la communauté scientifique face à la théorie du prion (Keyes, 1999).

S'il nous semble important de ne pas mélanger l'ordre des idées religieuses et celui des idées scientifiques, le principe de l'opposition montre certaines limites en ce qu'il donne une légitimité scientifique au créationnisme. On a alors l'impression de trouver une opposition théorique de plus dans le champ de la connaissance. Ces oppositions se retrouvent régulièrement dans l'histoire des idées, et l'on pourrait citer les plus célèbres, comme les oppositions vitalisme/mécanisme, inné/acquis, 
empirisme/intellectualisme... Si le débat inné/acquis a fini par trouver la solution d'une phénoménologie du sujet dans l'émergence du constructivisme, en dépassant l'opposition entre un individu placé dans une tour d'ivoire et le monde extérieur défini objectivement, toutes les oppositions n'ont pas connu une fin si heureuse (si tant est que le débat ait réellement pris fin). Notamment, l'opposition entre le vitalisme et le mécanisme semble s'être soldée par la victoire pure et simple du mécanisme cartésien et du matérialisme leibnizien. Or, comme le fait remarquer Canguilhem (1965c) dans son ouvrage La Connaissance de la vie, lorsque l'un de ces différents pôles opposés prend le dessus sur un autre, le «perdant » ne disparaît pas de l'histoire des idées. Il s'endort pendant un temps plus ou moins long, jusqu'à ce que la vision qu'il propose retrouve une certaine pertinence et fasse sens à nouveau. Et là se trouve peutêtre le risque de donner au créationnisme le statut d'une théorie alternative de plus à la théorie synthétique de l'évolution et de le voir revenir tôt ou tard sur le devant de la scène. De fait, une discussion philosophique nous semble plus à même de clarifier le débat qu'une discussion scientifique.

Une voie nous paraît essentielle à mettre en parallèle à celle de l'opposition entre créationnisme et théorie synthétique : celle du questionnement interne de la pratique scientifique. Cela appelle à ne pas uniquement se focaliser sur ce qui est vu comme des points d'erreurs de l'autre théorie et sur ce qui fait le propre de la science, mais à regarder les points de surgissements où la science fait des entorses à ses propres convictions (lorsque Crick invente le "dogme central», par exemple). L'ostracisme de la pure opposition entre créationnisme et théorie synthétique pourrait fort bien amener inversement à un certain communautarisme, à un conservatisme vis-à-vis de notre propre manière de considérer le monde et à une critique émoussée de nos propres apories théoriques. Ce qui s'illustre dans tout ce débat créationnisme/théorie synthétique, c'est la nécessité pour les scientifiques de ne pas être uniquement des utilisateurs de concepts, mais également des philosophes qui réfléchissent sur les concepts qu'ils utilisent et sur leur histoire. En cela la contribution de Kathryn Tabb (2007) à ce forum est particulièrement illustrative de cette approche. Selon elle, l'hésitation des scientifiques à utiliser la téléologie et à souligner le rôle qu'elle a pu avoir, notamment dans le développement et la pratique de la théorie évolutionniste, aurait ouvert la voie aux partisans de l'Intelligent Design pour utiliser la métaphore de Darwin à leur avantage. Nous proposons ici des éléments de réflexion sur l'ambiguïté de la notion de téléologie au travers des apports du courant philosophique de la phénoménologie. Cela amène notamment à reconsidérer la proximité des notions de vie utilisées par la religion et la science.

\section{Une certaine mécanisation de la vie}

Si, comme l'a rappelé K. Tabb (ibid.), l'image de la montre tient lieu d'argument à William Paley et au créationnisme pour évoquer le doigt du Créateur et justifier le recours à la téléologie, c'est en référence au même objet que Descartes a fondé son idée de l'animal-machine. C'est justement cette mécanisation du vivant que l'on retrouve avec la thématisation de la téléonomie en biologie (Pittendrigh, 1958 ; Mayr, 1961 et 1965 ; Monod, 1970) pour remplacer le concept de la téléologie, comme cela a pu être le cas dans le cadre de la théorie de l'évolution et dont on retrouve l'application en éthologie. Il n'y aurait aucun dessein intelligent dans la Nature, mais l'on pourrait avoir l'impression d'en voir un (cela serait « comme si ») dans l'expression d'un "programme génétique » conservé par la sélection naturelle (Monod, 1970). C'est au travers de la recherche de cette loi de la Nature, de ce voнoб de la téléonomie que l'on pourrait espérer éliminer la notion de cause finale de la téléologie.

Toutefois, comme K. Tabb (2007) l'a très justement rapporté, la notion de cause finale propre à la téléologie a été traitée de différentes manières dans l'histoire des idées, depuis la pensée aristotélicienne jusqu'à la métaphore de la «main invisible » d'Adam Smith, dont on retrouve l'influence dans les écrits de Darwin. Elle cite ainsi l'analyse de James Lennox et la distinction qu'il fait entre différents types de téléologie selon la position de «l'agent » qui serait à l'œuvre et laissant à penser qu'une finalité existe. Dans certaines approches, cet « agent » serait à l'intérieur du système décrit, comme c'est le cas pour la théorie synthétique de l'évolution ou dans les théories objectivistes du comportement. «L'agent » ne serait alors pas nécessairement un être physique, mais pourrait également être un terme métaphorique (la «main invisible») utilisé pour décrire ce que l'on appellerait désormais une sorte de propriété émergente du système. Ce qui caractérise la téléologie utilisée par les approches créationnistes, c'est le caractère externe de l'agent par rapport au système pris en compte. À moins de se placer dans une posture panthéiste comme celle de Spinoza, l'attitude de la religion pose toujours la déité à l'extérieur du monde (un peu comme l'observateur dans les approches positivistes de la science pour faire un aparté).

L'élimination de la téléologie des études du comportement devait permettre de suppléer au recours à la subjectivité, ce que l'on retrouve dans les écrits de Mayr (1961 et 1965) ainsi que dans ceux de Tinbergen (1963) - le recours à la physiologie permettant, selon les propres termes de Tinbergen, de remonter le continuum de la biologie moléculaire au comportement. Il donne ainsi une voie d'accès à la cause première du comportement sans mettre en jeu une quelconque volonté de l'individu. Cette approche mécaniste du comportement a été choisie très explicitement par Lorenz (1950) en opposition au 
vitalisme de son époque. On retrouve cette idée de nonnécessité de recours à l'intériorité de l'animal qu'autorise l'approche mécaniste dans le projet de naturalisation de l'esprit (Petitot et al., 2002).

\section{Où est la vie?}

Cette approche mécaniste et «physicaliste » n'est pas qu'une manière de questionner le vivant, mais va fonder le statut de l'individu que la pratique scientifique se donne pour tâche de connaître. Une des questions centrales levées par cette approche va être de se demander quelle est la spécificité du vivant. Pour Berryman (2003), le vivant pourrait tout à fait ne pas avoir besoin de ses lois de la Nature, celles-ci n'étant que des extensions au vivant de lois de la Physique. Quid, dans ces conditions, de ce qui distingue le vivant de la matière et l'animal dit "supérieur ", du végétal ou de l'amibe? Ce qui importe, c'est la loi sous-jacente au comportement et non pas ce qui fait sens pour l'individu. L'extension du raisonnement à une gamme particulièrement large d'êtres est d'ailleurs révélatrice de cette réification du statut de l'individu, voire, plus largement, du vivant.

Ce changement de statut du vivant, qui va de pair avec l'énoncé de la $v o \mu \sigma \sigma$ sous la forme d'un rapprochement de l'animal et du végétal, n'est pas sans rappeler la notion grecque de $\zeta \omega \eta$ et crée une démarcation paradoxale de celle de $\beta \iota \iota \zeta$, qui est pourtant censée être au centre de bien des approches. L'éthologie s'est ainsi constituée littéralement comme une biologie du comportement (Tinbergen, 1963), et l'écologie comportementale, qui en a récupéré les concepts de base, devrait y souscrire également. Alors que le terme $\zeta \omega \eta$ ainsi que les autres termes qui dérivent du thème $\zeta \omega \omega$ parlent de la vie, ils se démarquent pourtant de ceux trouvant leur origine dans la racine $\beta \iota \zeta$. $\zeta \eta v$ et $\zeta \omega o v$ vont décrire des êtres vivants : pas seulement des animaux, mais aussi des végétaux, voire des hommes (Chantraine, 2000). Il en ressort que ce terme $\zeta \omega \eta$ décrit tout être doté d'une âme, et donc également les plantes, qui en sont dotées dans la pensée aristotélicienne. La notion de $\zeta o \tau \alpha$ désigne dans un poème de Théocrite la propriété d'être vivant (ibid.) et l'on ne peut que souligner la proximité entretenue avec un «principe vital » propre aux théories vitalistes. La notion de $\beta \iota \zeta \zeta$ et le verbe $\beta \iota \omega v \alpha \iota$ dont elle dérive renvoient, eux, à une manière de vivre en particulier, au fait de vivre de telle ou telle façon. Le terme n'est pas appliqué aux plantes, mais essentiellement à l'homme et quelquefois aux animaux. On retrouve ici une notion du vivant abordée par Canguilhem (1965c) pour décrire le vivant infra-humain et qui semble faire abstraction de la plante et de l'animal non mobile. L'individu est alors un "centre » autour ou à partir duquel rayonne son activité, cette activité créant un milieu qui prend du sens pour cet individu. Cela fait de ce vivant un être apportant ses propres normes, sans pour autant souscrire aux théories vitalistes (par exemple la conception de la "volonté » chez Schopenhauer). Une des rares formes nominales du verbe, $\beta \iota \omega \sigma \iota \sigma$, renvoie ainsi à une « manière de vivre » et $\beta \iota \tau$ d'être vécu » (Chantraine, 2000). L'idée sous-jacente est donc plus dans le registre du vécu et, pourrait-on dire, de l'existential que dans la description d'un processus du vivant. Le terme de biographie illustre plutôt bien cette idée. Il ne parle pas de l'écriture de la vie en général, mais au contraire de celle d'une vie très particulière, de ce qui fait sens pour cette vie et comment cette vie elle-même est un sens, illustre une manière d'être et souvent un engagement. On retrouve cette idée chez Buytendijk (1958), pour qui « l'organisme animal et humain ne fait pas que vivre, il existe, c'est-à-dire qu'il crée une relation avec l'entourage». Cette notion de relation est essentielle ici et se place en opposition avec les approches ne parlant du vivant $\mathrm{qu}^{\prime}$ en termes d'interaction avec son environnement. La notion d'interaction s'insère dans un cadre mécaniste et réaliste, celui de la causalité et du lien entre le stimulus et la réponse (Merleau-Ponty, 1942). Développé sous l'égide de la condition ceteris paribus, l'individu devient alors un élément réactionnel dans un système expérimental. La notion de relation s'insère, elle, dans une phénoménologie, c'est-à-dire dans une philosophie du sujet pour qui les choses prennent une apparence. Or, c'est précisément à l'acception de processus du vivant plutôt que d'existence que la biologie fait traditionnellement référence, ce qui est particulièrement perceptible lorsque les plantes et les animaux, dont les animaux dits "supérieurs », sont regroupés dans une même ontologie. Lamarck et Treviranus, en 1802, rassemblaient déjà les plantes et les animaux sous le terme général «d'être vivant », que l'on retrouve dans l'étymologie de la philosophie zoologique de Lamarck (Klein, 1980). McLaughlin (2002) rapporte que Hanov publie, entre 1762 et 1768, un ouvrage intitulé Natural Philosophy or Dogmatic Physics, qui mentionne la biologie comme une science fondatrice préliminaire traitant des lois communes à la botanique et à la zoologie. Claude Bernard, cité par Canguihem (1965c), procède de même en écrivant ses Leçons sur les phénomènes de la vie communs aux animaux et aux végétaux (1878). La racine $\beta \iota$ ऽ de «biologie » semble ainsi étymologiquement impropre, contrairement à l'utilisation qui en est faite pour «biographie ». Dans son article de 2002, Peter McLauglin rapporte également qu'avant l'usage consacré aux sciences du terme «biologie», celuici pouvait prendre le sens de «biographie», notamment lors d'une oraison funèbre. Au lieu d'être écrite, la vie particulière était racontée, ou plutôt déclamée dans un discours, ce qui correspond scrupuleusement à l'étymologie grecque de ce mot ( $\beta \iota \iota_{\text {: }}$ vie qualifiée, manière d'être, et $\lambda \circ \gamma o \sigma:$ discours).

Le point de départ de ce débat se situait sur le rapprochement des discours religieux et scientifiques. Il est 
frappant de constater que les notions de vie utilisées par la science et la religion sont, de fait, souvent étonnamment proches. Quand les hautes instances cléricales demandent de lutter contre l'avortement au nom de la protection de la vie, c'est la vie en général qui est mentionnée, peut-être la vie humaine, mais une vie qui ne relève pas du particulier, d'un existential. Lorsque Richard Dawkins (2000), dans un ouvrage de vulgarisation, se fait le chantre des espèces menacées d'extinction en arguant qu'une espèce a plus d'importance qu'un individu, quand bien même il ne resterait qu'un seul individu dans cette espèce, c'est la même notion de vie qui est prise en compte. La même argumentation a pu être formulée par Rendtorff et Kemp (2000) pour justifier le prix de la vie en général par la durée du temps évolutif nécessaire à la mettre en place, ce qui est surprenant d'un point de vue ontologique.

\section{Vies et téléologies : dépasser certaines oppositions}

Dans son article fondateur de 1963 présentant l'éthologie comme "l'étude biologique du comportement", Tinbergen décrit le comportement comme un trait de l'organisme, au même titre qu'un organe, reprenant en cela l'image donnée par Lorenz. Cette approche lui a alors permis de fonder l'étude de la causalité du comportement dans les termes d'une physiologie du comportement. Cet angle d'approche aurait dû permettre, selon Tinbergen, de mettre un terme à l'utilisation de la subjectivité (en tant que vécu expérientiel) dans l'explication du comportement et donc à la téléologie. Cette physiologie du comportement s'inscrivait directement dans l'étude d'une $\zeta \omega \eta$, même si, comme cela vient d'être dit, l'extension du discours mécaniste tend à faire sortir cette approche du domaine de la vie. De son côté, Buytendijk, dans son ouvrage L'Homme et l'animal (1958), définit le comportement comme une "manière d'être » et le fait correspondre à « un rapport, c'est-à-dire à une relation intelligible avec quelque chose ». Dans cette approche, le terme de subjectivité change. Il n'est plus seulement vu comme un vécu expérientiel propre à un individu, mais décrit également la capacité à se construire un paysage de sens au travers de son activité, comme cela est abordé par les études phénoménologiques continentales, bien que cela ne soit pas toujours étendu à l'animal (chez Heidegger notamment). Cette phénoménologie du comportement, en s'intéressant à une manière d'être d'individus particuliers, s'inscrit bien, elle, dans le domaine de la vie en tant que $\beta \iota \zeta_{\zeta}$. Les apports du courant philosophique de la phénoménologie et ses développements existentialistes ont ainsi permis d'initier un mouvement pour sortir de certaines oppositions. La Structure du comportement de Merleau-Ponty (1942) se pose justement comme une réflexion sur certaines de ces oppositions (par exemple entre l'empirisme et l'intellectualisme ou entre l'inné et l'acquis) et comment les dépasser. Cela a contribué à dynamiser la réflexion scientifique, notamment dans le champ des sciences cognitives, et permis l'émergence de théories et de concepts nouveaux, comme la théorie de l'énaction (Varela, 1988; Varela et al., 1993 ; Engel et König, 1993 ; Engel et al., 2001).

\section{Conclusions}

Cet apport de la phénoménologie à l'étude du comportement permet une nouvelle conceptualisation de la notion de téléologie qui s'accommode fort bien de la distinction faite par Lennox (1992) entre la téléologie interne et la téléologie externe. Cela ne revient pas à éradiquer l'utilisation de ce concept faite par l'approche créationniste, mais cela permet tout au moins de resituer des cas pour lesquels il peut être employé : ceux positionnant l'agent ayant une fin à l'intérieur du système étudié. Cette nouvelle conceptualisation de la téléologie permet d'enrichir l'appréhension de la notion de vie utilisée dans les sciences de la Nature, et peut-être justement de dépasser les difficultés inhérentes à l'assimilation de formes de vie radicalement différentes sous une approche commune. En soi, cette confusion entre les différentes notions de vie que l'on peut avoir en grec, et que l'on retrouve en allemand avec les termes Leben et Dasein, pose déjà un certain nombre de problèmes épistémologiques. Quelle connaissance du vivant avons-nous, si nous l'assimilons à du non-vivant? Ne risquons-nous pas de passer à côté de l'essentiel ? Cela pose également des problèmes autres qu'épistémologiques, qui ressortent de la sphère de la réflexion éthique. $\mathrm{Si}$, comme le revendique une pression sociale de plus en plus forte, il convient d'initier une réflexion éthique sur notre rapport à l'animal et sur son utilisation, mais que par ailleurs celui-ci est pensé par la Science comme une mécanique ou comme une plante, cela soulève quelques incohérences...

Considérer uniquement le débat créationnisme/ darwinisme comme une opposition d'écoles, de points de vue, et se livrer à un ostracisme visant à montrer en quoi l'autre a tort n'est pas très satisfaisant et peut même être contre-productif, en donnant une légitimité au créationnisme. Un des bénéfices que l'approche scientifique de la vie pourrait retirer de ce débat consisterait à regarder aussi bien les différences par lesquelles elle se démarque de la religion que ce qui l'en rapproche et qui constitue par là de réels problèmes épistémologiques. Cela pourrait amener à reconsidérer des travaux comme ceux de Merleau-Ponty, qui ont justement permis de dépasser le manichéisme d'anciennes oppositions (inné/acquis, mécanisme/vitalisme, empirisme/intellectualisme...). Nous avons essayé de le faire valoir dans ce texte, le recours

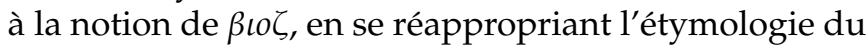


mot biologie, ouvre une porte sur un champ de recherche sur l'individu que la pratique classique de la science ignorait, et permet de lever certaines des ambiguïtés liées à l'utilisation du concept de téléologie.

\section{Références}

Berryman, A.A., 2003. On principles, laws and theory in population ecology, OIKOS, 103, 695-701.

Buytendijk, F.J.J., 1958. Mensch und Tier : ein Beitrag zur vergleichenden Psychologie, Hamburg, Rowohlt.

Canguilhem, G., 1965a. «L'expérimentation en biologie animale ", in Canguilhem, G., La Connaissance de la vie, Paris, Vrin, 19-49.

Canguilhem G., 1965b. « La théorie cellulaire », ibid., 53-101.

Canguilhem, G., 1965c. « Aspects du vitalisme », ibid., 105-127.

Chantraine, P., 2000. Dictionnaire étymologique de la langue grecque : histoire des mots, Paris, Klincksieck.

Crick, F.H.C., 1958. On protein synthesis, Symposium of the Society of Experimental Biology, 12, 139-163.

Crick, F., 1970. Central dogma of molecular biology, Nature, 227, 8, 561-563.

Dawkins, R., 2000. In Wolfe, A. [photographies], Conway, W. Dawkins, R., Goodall, J., et al. [texte], Libres et sauvages, Paris, Nathan, 89-91.

Engel, A.K., König, P., 1993. Paradigm shifts in neurobiology: Towards a new theory of perception, in Casati, R., White, G., Philosophy and the Cognitive Sciences, 131-138.

Engel, A.K., Fries, P., Singer, W., 2001. Dynamic predictions: Oscillations and synchrony in top-down processing, Nature Reviews Neuroscience, 2, 10, 704-716.

Feyerabend, P., 1988. Contre la méthode : esquisse d'une théorie anarchiste de la connaissance, Paris, Le Seuil.

Fridlansky, F., Mounolou, J.-C., 2007. Pourquoi dire «non» au « créationnisme »? (dossier " Évolution et créationnisme »), Natures Sciences Sociétés, 15, 4, 403-404.

Keyes, M.E., 1999. The prion challenge to the "Central Dogma" of molecular biology, 1965-1991. Part I: Prelude to prions, Stud. Hist. Phil. Biol. Biomed. Sci., 30, 1, 1-19.

Klein, M., 1980. Regards d'un biologiste. Évolution de l'approche scientifique : l'enseignement médical strasbourgeois, Paris, Hermann.

Latour, B., 1995. La Science en action, Paris, Gallimard.
Lennox, J.G., 1992. Teleology, in Keller, E.F., Lloyd, E.A. (Eds), Keywords in Evolutionary Biology, Cambridge, Harvard University Press, 323-333.

Lorenz, K., 1950. Trois essais sur le comportement animal et humain, Paris, Le Seuil.

Mayr, E., 1961. Cause and effect in biology: kinds of causes, predictability, and teleology are viewed by a practicing biologist, Science, 134, 3489, 1501-1506.

Mayr, E., 1965. Cause and effect in biology, in Lerner, D. (Ed.), Cause and Effect, New York, Free Press, 33-50.

McLaughlin, P., 2002. Naming Biology, Journal of the History of Biology, 35, 1, 1-4.

Merleau-Ponty, M., 1942. La Structure du comportement, Paris, PUF.

Merleau-Ponty, M., 1997. Parcours : 1931-1951, Paris, Verdier.

Monod, J., 1970. Le Hasard et la nécessité : essai sur la philosophie naturelle de la biologie moderne, Paris, Gallimard.

Pestre, D., 2006. Introduction aux «Sciences Studies », Paris, La Découverte.

Petitot, J., Varela, F.J., Pachoud, B., Roy, J.M., 2002. Combler le déficit : introduction à la naturalisation de la phénoménologie, in Naturaliser la phénoménologie : essais sur la phénoménologie contemporaine et les sciences cognitives, Paris, CNRS Éditions, $1-100$.

Pittendrigh, C.S., 1958. Adaptation, natural selection and behaviour, in Roe, A., Simpson, G.G. (Eds), Behavior and Evolution, New Haven (CT), Yale University Press, 390-419.

Prigogine, I., Stengers, I., 1979. La Nouvelle Alliance : métamorphose de la science, Paris, Gallimard.

Rendtorff, D. J., Kemp, P., 2000. Basic Ethical Principles in European Bioethics and Biolaw, vol. I. Autonomy, Dignity, Integrity and Vulnerability. Report to the European commission of the Biomed-II project, basic ethical principles in bioethics and biolaw 1995-1998, Centre for ethics and law, Copenhagen, Denmark / Institut Borja de bioética, Barcelona, Spain.

Tabb, K., 2007. La téléologie de Darwin (dossier « Évolution et créationnisme »), Natures Sciences Sociétés, 15, 3, 287-290.

Tinbergen, N., 1963. On aims and methods of ethology, Zeitschrift für Tierpsychologie, 20, 410-433.

Varela, F., 1988. Connaître : les sciences cognitives, tendances et perspectives, Paris, Le Seuil.

Varela, F., Thompson, E., Rosch, E., 1993. L'Inscription corporelle de l'esprit : sciences cognitives et expérience humaine, Paris, Le Seuil. 